\title{
Acute epiploic appendagitis: ultrasound and computed tomography findings of a rare case of acute abdominal pain and the role of other imaging techniques
}

\author{
Piero Trovato ${ }^{1}$, Igino Simonetti ${ }^{1}$, Francesco Verde ${ }^{1}$, Pascal Lomoro ${ }^{2}$, Giorgia Vinci ${ }^{3}$, Luca Tarotto ${ }^{1}$, Fabio Corvino ${ }^{4}$, \\ Antonio Corvino ${ }^{5}$
}

'Department of Advanced Biomedical Sciences, University of Naples "Federico II', Naples, Italy

${ }^{2}$ Department of Radiology, Valduce Hospital, Como, Italy

'Department of Nephrology, University of Campania "Luigi Vanvitelli", Naples, Italy

${ }^{4}$ Department of Interventional Radiology, AORN “A. Cardarelli", Naples, Italy

${ }^{5}$ Department of Motor Science and Wellness, University of Naples “Parthenope”, Naples, Italy

\section{Abstract}

Purpose: Acute epiploic appendagitis (EA) is a relatively rare, benign and local inflammatory disease involving the epiploic appendices. Unlike its mimics, EA is generally a self-limiting inflammatory disease and can be treated conservatively.

Case presentation: A 33-year-old Caucasian man presented to our emergency department with a sever and sharp left iliac fossa pain. He underwent abdominal X-ray, ultrasound (US) and computed tomography (CT) evaluations.

Conclusion: We illustrate US and CT findings to increase the radiologists' awareness of this condition and to avoid diagnostic delay and unnecessary use of antibiotics, hospitalization and surgery.

Key words: epiploic appendagitis, abdominal pain, acute abdomen, computed tomography (CT), ultrasound (US), emergency radiology.

\section{Introduction}

Epiploic appendagitis (EA), also known as appendicitis epiploicae, epiplopericolitis, or appendagitis, is a relatively rare, benign, and local inflammatory disease involving the epiploic appendices [1-4].

The epiploic appendices (or epiploic appendage or omental appendices) are peritoneal outpouchings generally located in two rows adjacent to the anterior and posterolateral taenia coli, characterised by adipose tissue, one or two arterioles that branch from the vasa recta longa of the colon, and a single draining venule [2,5-8]. Their role is not well known; it has been proposed that they can have a protective function towards intestinal vessels during the processes of distension or collapse of the colon $[2,5]$.

These pedunculated omental fat protrusions have a normal thickness of 1-2 cm and a medium length of 3 $\mathrm{cm}$ (range between $5 \mathrm{~mm}$ and $5 \mathrm{~cm}$ ), with the largest ones generally distributed adjacent to the sigmoid colon, and are located from the cecum to the recto-sigmoid in a number of 50-100 in most adults, not localising however near the rectum [9-13].

The term "epiploic appendagitis" was initially described in 1956 by Lynn et al., and its computed tomography (CT) features were described for the first time in 1986 by Danielson et al. [12,14-16].

Correspondence address:

Igino Simonetti, MD, Department of Advanced Biomedical Sciences, University of Naples "Federico II", Via Sergio Pansini, 5, 80131 - Naples, Italy,

e-mail: igino.simonetti@gmail.com

Authors' contribution:

A Study design - B Data collection - C Statistical analysis - D Data interpretation - E Manuscript preparation - F Literature search - G Funds collection 
This disease typically affects people aged $20-50$ years, with a greater frequency in men than in women $(4: 1)$ [1,17-20].

Risk factors include male gender, obesity, intense exercise, colonic diverticula, and hernias $[8,12]$. EA can be primary or secondary. Primary EA is an inflammatory disease that may arise from a spontaneous torsion causing obstruction of blood flow within the omental appendage, then ischaemia up to a necrosis, or from spontaneous thrombosis of the draining vein and inflammation $[13,15,21]$. Instead, secondary EA may arise from adjacent inflammatory diseases involving the colonic wall and surrounding mesocolon, such as diverticulitis or appendicitis $[1,12]$.

Thomas et al. reviewed 197 cases from the literature and 11 of their cases of EA, and classified each according to its cause: torsion and inflammation (73\%), hernia incarceration (18\%), intestinal obstruction (8\%), and intraperitoneal loose body (1\%) [12,22].

The most common sites involved by this disease are the rectosigmoid (57\%) and the ileocecum (26\%); rarer sites are the ascending (9\%), transverse $(6 \%)$, and descending colon $(2 \%)[8,9,12]$. Clinically, EA manifests in most cases (60-80\%) with acute or subacute abdominal pain in the left lower quadrant, but it can also involve the right lower quadrant, thus miming other diseases such as appendicitis, diverticulitis, acute cholecystitis, and omental infarction $[12,23,24]$. However, most patients have a normal body temperature, and laboratory results generally show a normal white blood cell (WBC) count [12,25]. In fewer cases, patients also complain of diarrhoea and constipation [23].

Unlike its mimics, EA is a self-limiting inflammatory disease in most patients, with an average of 10 days, and can be treated conservatively, only with anti-inflammatory medication $[1,3,11]$; rarely it may cause the development of adhesion, bowel obstruction or intussusception, intra- peritoneal loose body (peritoneal "mice"), peritonitis, and abscess $[1,11]$. For these reasons, it is very important to differentiate EA from the other diseases causing abdominal pain, such as acute appendicitis, which usually require surgery $[1,8]$.

Today, ultrasound (US) and CT (preferred) scans play a crucial role in the diagnosis of this disease [12,26-28].

We present a rare case of epiploic appendagitis with the purpose of increasing awareness of this condition as a cause of acute-subacute abdominal pain and knowledge of its US and CT features, in order to avoid a diagnostic delay and unnecessary use of antibiotics, hospitalisation, and surgery.

\section{Case presentation}

A 33-year-old Caucasian male presented to our emergency department with a sever and sharp left iliac fossa pain that was sore on inspiration, coughing, and walking, which had started the day before. He denied nausea, vomiting, fever, alteration of intestinal habits, trauma to the area, dysuria, haematuria, loss of weight, or skin rash. Surgical history was negative and without chronic medications. Review of systems was otherwise negative. On abdominal examination he had localised tenderness in the left iliac fossa with guarding and rebound tenderness. There was no pulsatile or palpable mass, or costovertebral angle tenderness. Physical examination was otherwise unremarkable.

Therefore, the patient underwent laboratory and diagnostic tests. Laboratory results showed white blood cell (WBC) count of $14.10 \times 1000 / \mu \mathrm{l}(4.8-10.8)$ with neutrophilia (88.6\%).

Abdominal X-ray showed no pathological air-fluid levels and no free subphrenic gas (Figure 1). An evaluation with abdominal US was performed and revealed a reactive bowel wall thickening of the descending and
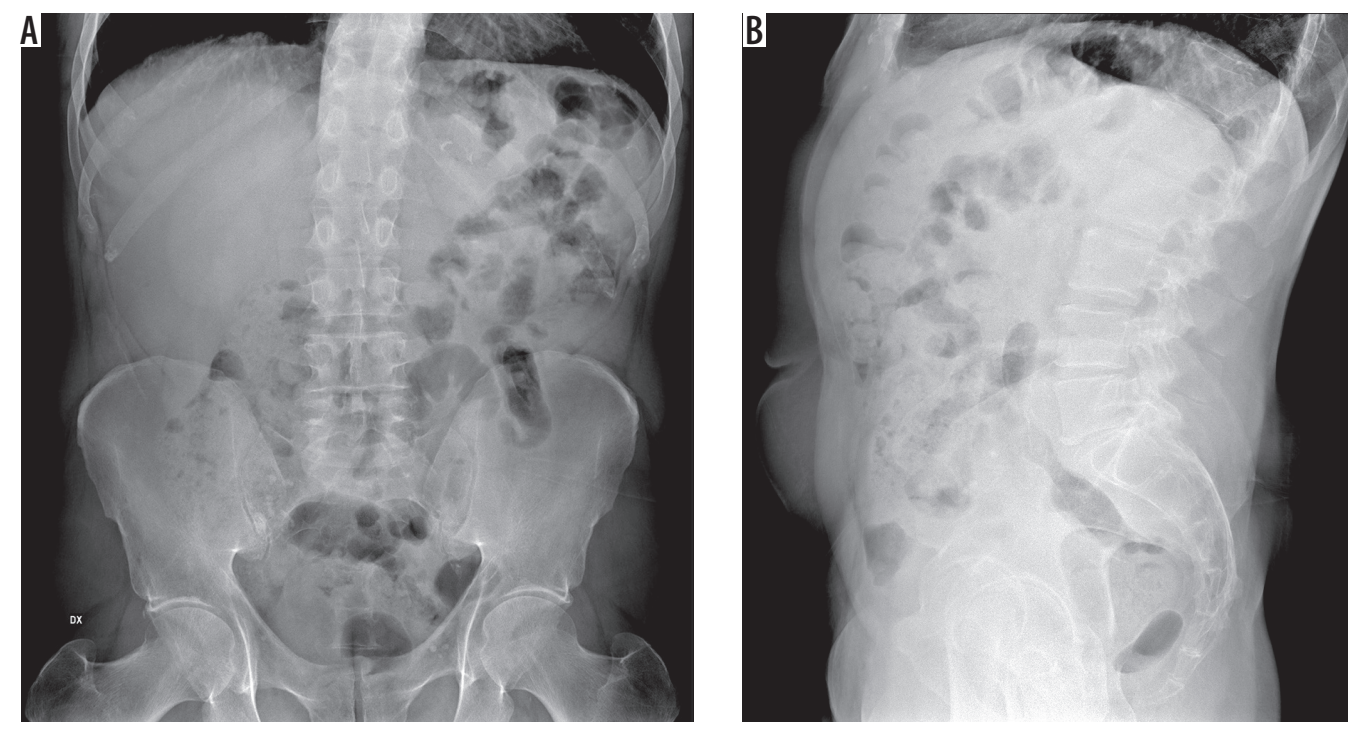

Figure 1. Abdominal X-ray showed no pathological air-fluid levels and no free subphrenic gas 

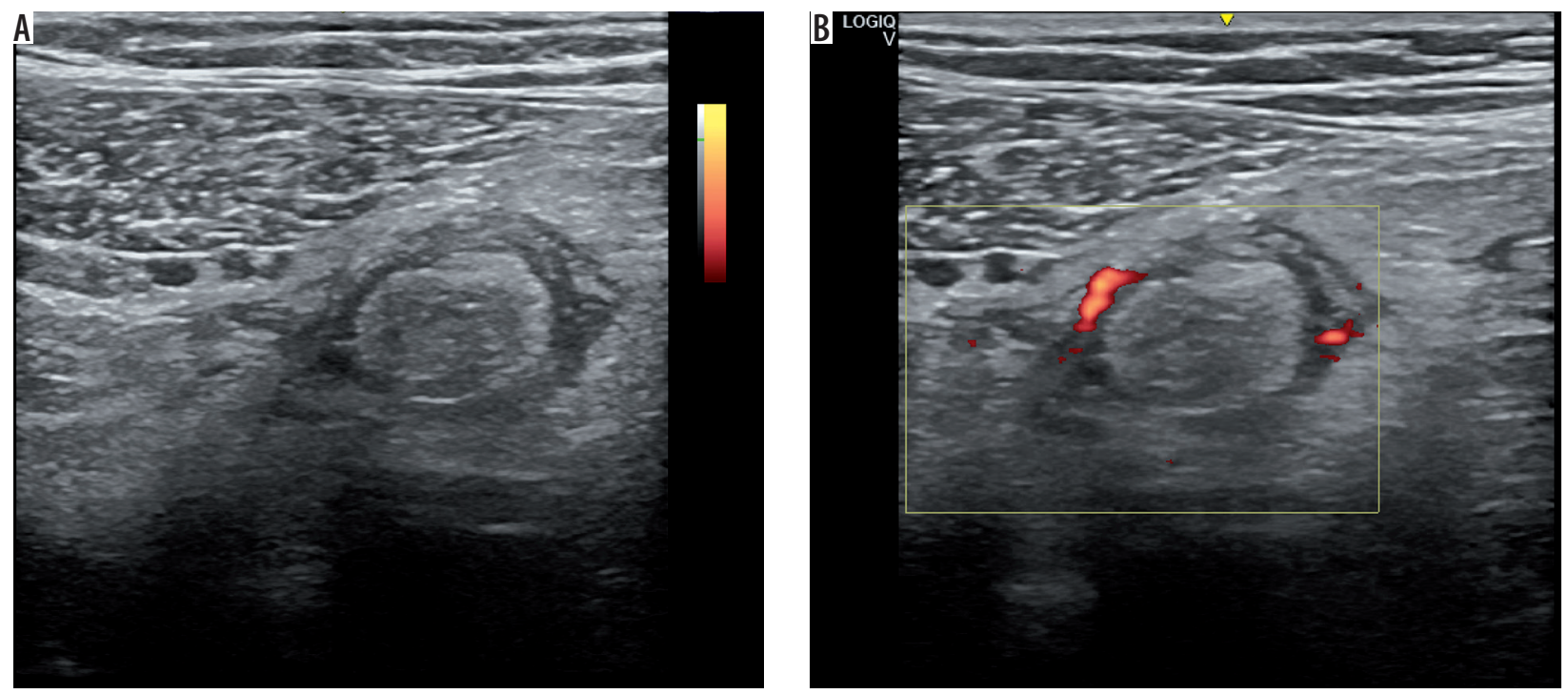

Figure 2. Abdominal US image of the left lower quadrant showed a reactive bowel wall thickening of the descending and the sigmoid colon with inflammatory changes in the pericolonic fat, which appeared as an adjacent, oval, non-compressible, hyperechoic mass, without internal vascularity, surrounded by a subtle hypoechoic line, and at least three perivisceral lymph node formations that were likely to be reactive

the sigmoid colon with inflammatory changes in the pericolonic fat, which appeared as an adjacent, oval, noncompressible, hyperechoic mass, without internal vascularity, surrounded by a subtle hypoechoic line and at least three perivisceral lymph node formations that were likely to be reactive (Figure 2). Abdominopelvic CT with intravenous and oral contrast agents was also performed and revealed a moderate reactive wall thickening of the descending and the sigmoid colon and a non-enhancing adjacent fat-density ovoid structure $\left(16^{\prime} 18^{\prime} 15 \mathrm{~mm}\right)$ with high-density rim and surrounding inflammatory fat stranding (Figure 3).

\section{Discussion}

EA is an uncommon cause of acute abdomen with a clinical presentation resembling other causes of acute abdominal pain such as diverticulitis and appendicitis. Indeed, before the widespread utilisation of modern diagnostic imaging techniques, in particular CT and US, the diagnosis of EA was usually made during surgical exploration while searching for unexplained cause of acute abdomen. Nowadays, although normal epiploic appendages are usually not evident in radiological studies, they result visible in inflammatory conditions and can be definitively diagnosed by contrast-enhanced CT (CECT) and US [3,29-31]. Moreover, other advanced imaging techniques can be useful in the diagnosis of this disease such as dual-energy CT (DECT), contrast-enhanced ultrasound (CEUS), and magnetic resonance imaging (MRI) [20,32].

The classic CECT findings of acute EA consist of a well-defined, rounded or ovoid fat-attenuation mass abutting the wall of the colon, of 2 to $5 \mathrm{~cm}$ in size, enveloped by a continuous higher attenuation rim (the "hyperattenuating ring") due to inflammation, which may occasionally involve the parietal peritoneum resulting in

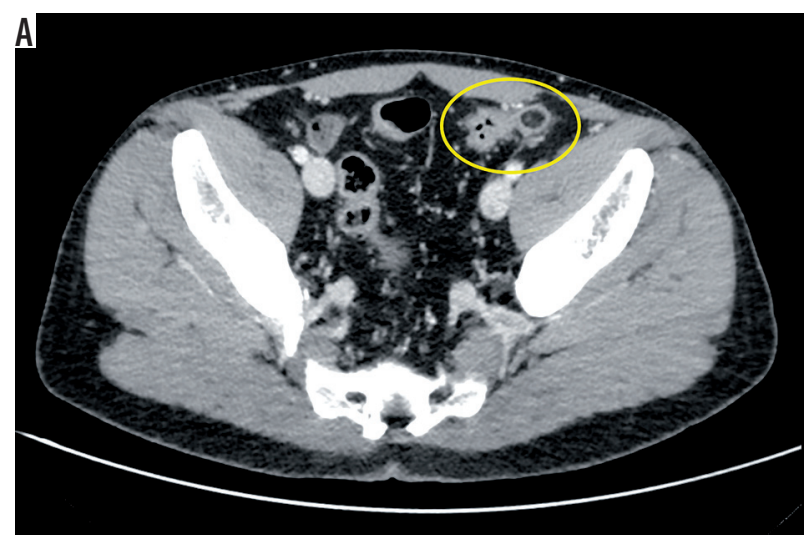

Figure 3. Abdominopelvic computed tomography with intravenous and oral contrast agents (axial and coronal scans) showed a moderate reactive wall thickening of the descending and the sigmoid colon and a non-enhancing adjacent fat-density ovoid structure with high-density rim and surrounding inflammatory fat stranding

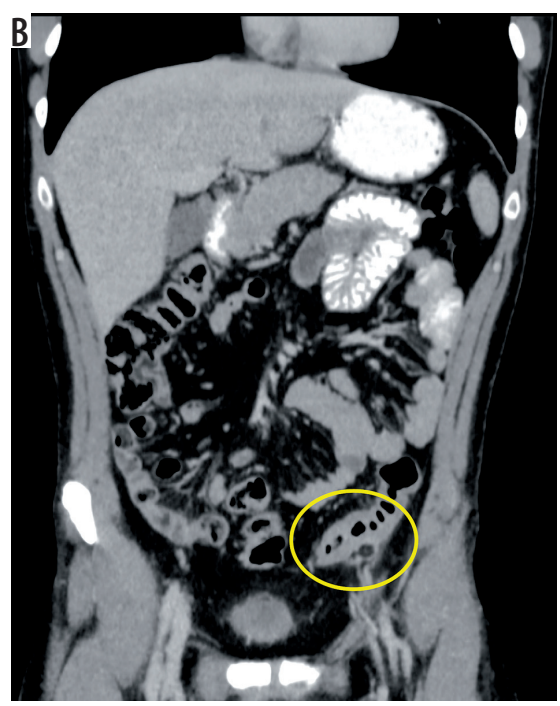


oedematous thickening appearances $[3,7,33]$. Acute EA can rarely cause thickening of the colonic wall or can be located in the hernia sac [34].

Another important CT feature, although not always visible, is the "central dot sign", a central high attenuation region correlated to a thrombosed draining appendageal vessel or to internal haemorrhage or fibrous tissue $[4,35,36]$. An epiploic appendage may completely be separated from its pedicle, resulting in a wandering fatty abdominal mass that can calcify $[7,12]$. Other rare complications of EA include peritonitis, adhesions, abscess formation, bowel obstruction, and intussusception [37].

Additionally, in a recent study regarding the application of DECT for gastrointestinal imaging, it was highlighted that in the case of EA there is a higher iodine uptake of the surrounding fat secondary to inflammation, and lack of iodine uptake within the infarcted epiploic appendage [38]. The advantage of this technique is the full field of view and application of dose modulation in both acquisitions, as well as greater sensitivity than conventional CT alone without additional radiation dose for the patient [38].

The characteristic finding of acute EA on US is a hyperechoic, ovoid, non-compressible mass with a greater diameter ranging from 2 to $4 \mathrm{~cm}$. The lesion is located at the site of maximum tenderness, usually under the abdominal wall, and is attached to the adjacent colonic wall. Moreover, it may show a peripherical hypoechoic rim due to thickening of the serosal covering of appendages and of the parietal peritoneum $[7,12]$. In colour Doppler US, acute EA shows absent or weak internal blood flow, in contrast to appendicitis or diverticulitis, which are abun- dantly vascularised [12]. The use of CEUS may be useful to confirm the diagnosis of EA in unclear cases. On CEUS, lesions show a central area of no enhancement with a variable thickness of perilesional enhancement (> $1 \mathrm{~cm}$ in most cases) [39].

Although magnetic resonance is not routinely performed, MRI findings of epiploic appendagitis appear to correlate with CT findings. In this regard, T1- and T2-weighted images show a focal lesion with the signal intensity of fat and a peripherical enhancing rim on postgadolinium T1-weighted images [40].

CECT imaging surely represent the method of choice to detect EA and exclude other acute abdominal diseases that which are considered in the differential diagnosis, such as acute appendicitis, acute diverticulitis, acute omental infarction, sclerosing mesenteritis, and tumour or metastasis to the mesocolon. For females, it is important to take into account also ovarian torsion, ovarian cyst rupture, and ectopic pregnancy [3].

\section{Conclusions}

Unlike its mimics, EA is a self-limiting inflammatory condition and generally requires conservative treatment $[3,7,12,20]$. For these reasons, radiologists' knowledge and awareness of EA as a cause for abdominal pain and its radiographic findings can prevent unnecessary hospitalisation and surgery [1,7].

\section{Conflict of interest}

The authors report no conflict of interest.

\section{References}

1. Chan E, El-Banna A. A case report of epiploic appendagitis as a mimic of acute cholecystitis. Int J Surg Case Rep 2018; 53: 327-329.

2. Pines B, Rabinovitch J, Biller SB. Primary torsion and infarction of the appendices epiploicae. Arch Surg 1941; 42: 775-787.

3. Patel H, Abdelbaki A, Steenbergen P, et al. Know the name: acute epiploic appendagitis - CT findings and review of literature. AME Case Rep 2018; 2: 8.

4. Giambelluca D, Dimarco M, Vaccaro Notte MR, et al. The "central dot sign" in acute epiploic appendagitis. Abdom Radiol (NY) 2019; 44: 1606-1607.

5. Plummer R, Sekigami Y, Chen L, et al. Epiploic appendagitis mimicking recurrent diverticulitis. Case Rep Surg 2018; 2018: 1924067.

6. Vinson DR. Epiploic appendagitis: a new diagnosis for the emergency physician. Two case reports and a review. J Emerg Med 1999; 17: 827-832.

7. Di Serafino M, Iacobellis F, Trovato P, et al. Acute epiploic appendagitis: a nonsurgical abdominal pain. Case Rep Emerg Med 2019; 2019: 7160247.

8. Chu EA, Kaminer E. Epiploic appendagitis: a rare cause of acute abdomen. Radiol Case Rep 2018; 13: 599-601.
9. Schnedl WJ, Krause R, Tafeit E, et al. Insights into epiploic appendagitis. Nat Rev Gastroenterol Hepatol 2018; 13: 599-601.

10. Perez-Girbes A, Alegre A, Linnau KF. Core curriculum illustration: epiploic appendagitis. Emerg Radiol 2020; 27: 221-222.

11. Lorente C, Hearne CB, Taboada J. Recurrent epiploic appendagitis mimicking appendicitis and cholecystitis. Proc (Baylor Univ Med Cent) 2017; 30: 44-46.

12. Singh AK, Gervais DA, Hahn PF, et al. Acute epiploic appendagitis and its mimics. Radiographics 2005; 25: 1521-1534.

13. Tabbara TA, Alassaf OY, Kaouas MC. Acute epiploic appendigitis: diagnostic and laparoscopic approach. Int J Surg Case Rep 2018; 44: 157-160.

14. Dockerty MB, Lynn TE, Waugh JM. A clinicopathologic study of the epiploic appendages. Surg Gynecol Obstet 1956; 103: 423-433.

15. Ergelen R, Asadov R, Özdemir B, et al. Computed tomography findings of primary epiploic appendagitis as an easily misdiagnosed entity: case series and review of literature. Ulus Travma Acil Cerrahi Derg 2017; 23: 489-494.

16. Danielson K, Chernin MM, Amberg JR, et al. Epiploic appendicitis: CT characteristics. J Comput Assist Tomogr 1986; 10: 142-143. 
17. Ross JA. Vascular loops in the appendices epiploicae; their anatomy and surgical significance, with a review of the surgical pathology of appendices epiploicae. Br J Surg 1950; 37: 464-466.

18. Ozdemir S, Gulpinar K, Leventoglu S, et al. Torsion of the primary epiploic appendagitis: a case series and review of the literature. Am J Surg 2010; 199: 453-458.

19. Nugent JP, Ouellette HA, O’Leary DP, et al. Epiploic appendagitis: 7-year experience and relationship with visceral obesity. Abdom Radiol (NY) 2018; 43: 1552-1557.

20. Simonetti I, Puglia M, Tarotto L, et al. When traditions become dangerous: intestinal perforation from unusual foreign body - case report and short literature review. Eur J Radiol Open 2019; 6: 152-155.

21. Golash V, Willson PD. Early laparoscopy as a routine procedure in the management of acute abdominal pain: a review of $1,320 \mathrm{pa}-$ tients. Surg Endosc Other Interv Tech 2005; 19: 882-885.

22. Thomas JH, Rosato FE, Patterson LT. Epiploic appendagitis. Surg Gynecol Obstet 1974; 138: 23-25.

23. Vaghela K, Shah B. Diagnosis of acute appendicitis using clinical Alvarado scoring system and computed tomography (CT) criteria in patients attending Gujarat Adani Institute of Medical Sscience a retrospective study. Pol J Radiol 2017; 82: 726-730.

24. Kamr WH, Taman SE, Tawfik AI. Multislice computed tomography evaluation of primary abdominal fat necrosis: a rare cause of acute abdominal pain. Pol J Radiol 2017; 82: 726-730.

25. Evrimler S, Okumuser I, Unal N. Computed tomography (CT) findings of a diagnostic dilemma: atypically located acute appendicitis. Pol J Radiol 2016; 81: 583-588.

26. Simonetti I, Trovato P, Verde F, et al. A rare case of hydrometrocolpos from persistent urogenital sinus in patient affected by adrenogenital syndrome. J Ultrasound 2018; 21: 249-252.

27. Corvino A, Catalano O, Corvino F, et al. Diagnostic performance and confidence of contrast-enhanced ultrasound in the differential diagnosis of cystic and cysticlike liver lesions. Am J Roentgenol 2017; 209: w119-w127.

28. Trovato P, Simonetti I, Verde F, et al. A rare case of intrahepatic abscess secondary to spontaneous gallbladder perforation in a cancer patient treated by interventional radiology procedure. J Radiol Rev 2019; 6: 45-49. doi: 10.23736/S2283-8376.19.00153-0.

29. Abu-Zidan FM, Cevik AA. Diagnostic point-of-care ultrasound (POCUS) for gastrointestinal pathology: State of the art from basics to advanced. World J Emerg Surg 2018; 13: 47.

30. Hasbahceci M, Erol C, Seker M. Epiploic appendagitis: is there need for surgery to confirm diagnosis in spite of clinical and radiological findings? World J Surg 2012; 36: 441-446.

31. Lomoro P, Simonetti I, Vinci G, et al. Pancake kidney, a rare and often misdiagnosed malformation: a case report and radiological differential diagnosis. J Ultrasound 2019; 22: 207-213.

32. Corvino A, Sandomenico F, Setola SV, et al. Morphological and dynamic evaluation of complex cystic focal liver lesions by contrast-enhanced ultrasound: current state of the art. J Ultrasound 2019; 22: 251-259.

33. Han Q, Nair RT, DiSantis DJ. The hyperattenuating ring sign of acute epiploic appendagitis. Abdom Radiol 2016; 41: 1431-1432.

34. Purysko AS, Remer EM, Filho HML, et al. Beyond appendicitis: common and uncommon gastrointestinal causes of right lower quadrant abdominal pain at multidetector CT. Radiographics 2011; 31: 927-947

35. Nejadhamzeeigilani $H$. The central dot sign of epiploic appendagitis. Abdom Radiol 2019; 44: 802-803.

36. Eberhardt SC, Strickland CD, Epstein KN. Radiology of epiploic appendages: acute appendagitis, post-infarcted appendages, and imaging natural history. Abdom Radiol 2016; 41: 1653-1665.

37. Kamaya A, Federle MP, Desser TS. Imaging manifestations of abdominal fat necrosis and its mimics. Radiographics 2011; 31: 20212034.

38. Ali IT, Thomas C, Elbanna KY, et al. Gastrointestinal imaging: emerging role of dual-energy computed tomography. Curr Radiol Rep 2017; 5: 31 .

39. Görg C, Egbring J, Bert T. Contrast-enhanced ultrasound of epiploic appendagitis. Ultraschall Der Medizin 2009; 30: 163-167.

40. Şirvanci M, Tekelioğlu MH, Duran C, et al. Primary epiploic appendagitis: CT manifestations. Clin Imaging 2000; 24: 357-361. 